Check for updates

Cite this: RSC Adv., 2018, 8, 3928

\title{
Many-body effects and non-local charge fluctuations in the double perovskite $\mathrm{Sr}_{2} \mathrm{FeMoO}_{6}$
}

Received 19th October 2017 Accepted 13th January 2018

DOI: $10.1039 / \mathrm{c} 7 \mathrm{ra11577f}$

rsc.li/rsc-advances

\author{
Henrique P. Martins, (D) *a Eduardo B. Guedes, ${ }^{a}$ Rodrigo J. O. Mossanek, \\ Fernando D. Prado, ${ }^{b}$ Alberto Caneiro ${ }^{c}$ and Miguel Abbate ${ }^{a}$
}

We studied the electronic structure of $\mathrm{Sr}_{2} \mathrm{FeMoO}_{6}$ using core level and valence band photoemission. The spectra were obtained using high energy $\mathrm{X}$-rays of $1840 \mathrm{eV}$, which provide bulk sensitive information on the electronic structure. The experimental data were analyzed using the spectral weight from cluster model calculations. The ground state reveals a large $\mathrm{Fe}-\mathrm{O}$ and Mo-O hybridization, as well as the importance of non-local Fe-O-Mo charge fluctuations. The latter is crucial to explain the half-metallic character attributed to the $\mathrm{Sr}_{2} \mathrm{FeMoO}_{6}$ compound. The core level and valence band photoemission spectra show charge transfer satellites. These satellites are related to many-body effects and are larger for the Fe levels than for the Mo states.

\section{Introduction}

The $\mathrm{Sr}_{2} \mathrm{FeMoO}_{6}$ (SFMO) double perovskite presents very interesting physical properties. ${ }^{\mathbf{1 , 2}}$ The crystal structure of this compound is tetragonal with $a=5.557 \AA$ and $c=7.887 \AA$. This material is a half-metallic ferrimagnet with an ordering temperature of $T_{\mathrm{C}}=420 \mathrm{~K}$. The magnetic moment, up to $\mu=3.7 \mu_{\mathrm{B}}$, depends on the degree of Fe/Mo cationic disorder. ${ }^{3}$ The SFMO oxide exhibits a considerable magneto-resistance (MR) at room temperature. The accepted MR mechanism involves tunneling across grain boundaries. For these reasons, this compound is being considered for applications in spintronic devices. More details on the SFMO double perovskite can be found in recent reviews. ${ }^{4-6}$

The electronic structure of SFMO was studied using a variety of spectroscopic techniques, which aimed at understanding the microscopic origin of its physical properties. ${ }^{7}$ The optical conductivity confirmed the half-metallic character of the SFMO compound. $^{2}$ The core-level X-ray photoelectron spectra (XPS) determined the chemical shifts of the Fe/Mo ions. ${ }^{\mathbf{8} 9}$ The valence band X-ray photoemission spectra (PES) elucidated the electronic states at the Fermi level..$^{10-12}$ The X-ray absorption spectra (XAS) was utilized to investigate the Fe/Mo valencies.9,11,13 The $\mathrm{X}$-ray emission spectra (XES) provided site selected information on the electronic structure. ${ }^{14}$ Finally, the magnetic circular dichroism spectra (MCD) was used to study the Fe/Mo magnetic moments. ${ }^{13,15}$

${ }^{a}$ Departamento de Física, Universidade Federal do Paraná, Caixa Postal 19044, 81531-990 Curitiba, PR, Brazil..E-mail:hpmartins@gmail.com

${ }^{b}$ Departamento de Física, Universidad Nacional del Sur, Av. Leandro N. Alem 1253, 8000 Bahía Blanca, Argentina

${ }^{c}$ Centro Atómico Bariloche, Comisión Nacional de Energía Atómica, Av. Ezequiel Bustillo 9500, 8400 Bariloche, Argentina
In an ionic approximation, the electronic structure of SFMO can be viewed as a combination of $\mathrm{Fe}^{3+}\left(3 \mathrm{~d}^{5}\right)$ and $\mathrm{Mo}^{5+}\left(4 \mathrm{~d}^{1}\right) .{ }^{1,2}$ However, the Fe $3 \mathrm{~d}$ and Mo $4 \mathrm{~d}$ electrons present a covalent hybridization with the $\mathrm{O} 2 \mathrm{p}$ states. ${ }^{12}$ Further, the majority $\mathrm{Fe}^{3+}$ $\left(3 \mathrm{~d}^{5}\right)$ electrons are localized, whereas the minority $\mathrm{Mo}^{5+}\left(4 \mathrm{~d}^{1}\right)$ electron is itinerant, which is consistent with both the halfmetallic conductivity and the ferrimagnetic ordering of SFMO. In fact, the conducting electron is delocalized along Mo-O-FeO-Mo chains in this material, indicating the importance of the Fe 3d-O 2p and Mo 4d-O 2p covalent mixing, as well as the nonlocal Mo-O-Fe charge fluctuations.

The valence band spectra of SFMO were compared to diverse density functional theory (DFT) methods. The agreement with the experiment improves if one includes in the calculation the Mott-Hubbard $\mathrm{U.}^{\mathbf{1 0 1 1}}$ The values of $U$ used in the LDA $+\mathrm{U}$ approach at the Fe sites are relatively large, about $U=2-4 \mathrm{eV}$. Based on these values, Kuepper et al. concluded that the electronic structure of SFMO was highly correlated. ${ }^{14}$ In particular, the valence band spectra of this oxide might present distinct many-body effects beyond the DFT description.

We studied the electronic structure of SFMO using a cluster model approach, ${ }^{\mathbf{1 6 - 1 9}}$ which consists of two $\mathrm{FeO}_{6}$ and $\mathrm{MoO}_{6}$ octahedra which are either connected (double cluster model) or not (single cluster model) by one corner $\mathrm{O}$ atom. This method includes both the Fe $3 \mathrm{~d}-\mathrm{O} 2 \mathrm{p}$ and Mo $4 \mathrm{~d}-\mathrm{O} 2 \mathrm{p}$ hybridization, as well as the Mo-O-Fe charge fluctuations in the $\mathrm{FeO}_{6}-\mathrm{MoO}_{6}$ double cluster. The electronic structure is calculated using the configuration interaction approach, ${ }^{\mathbf{1 7}}$ which includes many relevant correlation effects within the double cluster. The calculations are compared to the core level and valence band spectra of SFMO. Finally, the experimental spectra were obtained using bulk sensitive high-energy X-rays. 


\section{Experimental details}

The ceramic $\mathrm{Sr}_{2} \mathrm{FeMoO}_{6}$ sample was prepared using the solidstate reaction method. The corresponding reagents were mixed and calcined in air at $950{ }^{\circ} \mathrm{C}$ for $24 \mathrm{~h}$. The resulting powder was pulverized, mixed and fired again several times. The reduction of the powder was carried out in a flowing mixture of $1 \% \mathrm{H}_{2}-\mathrm{Ar}$ gas at $1050{ }^{\circ} \mathrm{C}$ for $1 \mathrm{~h}$. Finally, the substance was ground, pressed into pellets, and sintered in a vacuum for $12 \mathrm{~h}$ at $1200^{\circ} \mathrm{C}$. The powder XRD analysis confirmed that the sample was a single-phase material. The Rietveld refinement showed a tetragonal $14 / \mathrm{mmm}$ structure with the aforementioned parameters. The relative intensity of the (101) reflection indicated a highly ordered sample with less than $3 \%$ cationic disorder. $^{3}$

The photoemission measurements were performed at the SXS beamline at the Laboratório Nacional de Luz Síncrotron (LNLS) in Campinas (Brazil). ${ }^{20}$ The photon energy scale of the monochromator was calibrated using the Si K absorption edges. The photoemission spectra was taken using a SPECS Phoibos 150 electron energy analyzer; the energy scale of the analyzer was calibrated using a clean gold foil. All the spectra presented here were taken at room temperature with a photon energy of $1840 \mathrm{eV}$. The base pressure in the UHV experimental chamber was in the low $10^{-9}$ mbar range. The pellets were thoroughly scraped with a diamond file to remove the surface contamination. The core level Fe 2p and Mo 3p spectra presented here are new, whereas the valence band spectrum was already presented in a previous study. ${ }^{21}$

\section{Calculation details}

The electronic structure of $\mathrm{Sr}_{2} \mathrm{FeMoO}_{6}$ was calculated using a double cluster model, composed by an $\mathrm{FeO}_{6}$ and a $\mathrm{MoO}_{6}$ octahedra sharing a corner $\mathrm{O}$ atom. The double octahedra considered here is represented schematically in the top panel of

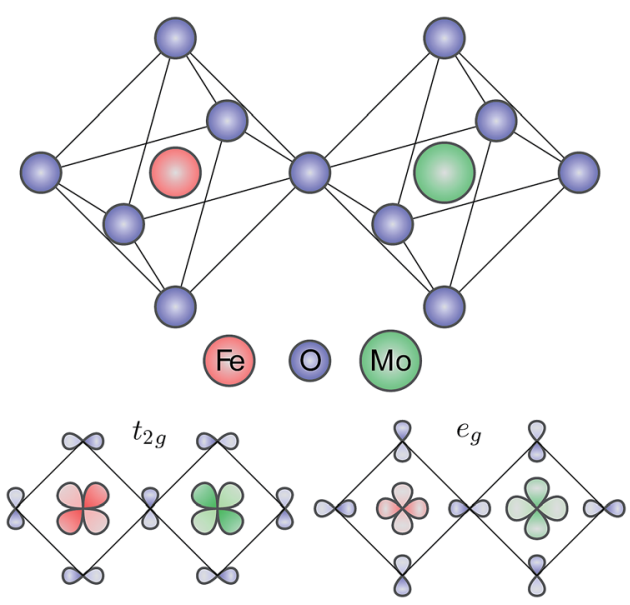

Fig. 1 Schematic representation of the octahedra used in the double cluster model calculation (top panel). Schematic representation of the symmetry-adapted combinations of orbitals with local $t_{2 g}$ and $e_{\mathrm{g}}$ characters (bottom panel).
Fig. 1. The double cluster model was solved using a symmetryadapted configuration interaction method. ${ }^{16,18}$ The Fe $3 \mathrm{~d}$ and Mo $4 \mathrm{~d}$ orbitals are split by crystal field effects into $t_{2 \mathrm{~g}}$ and $e_{\mathrm{g}}$ symmetries. The symmetry-adapted combination of orbitals with a local $t_{2 \mathrm{~g}}$ and $e_{\mathrm{g}}$ character are depicted in the bottom panel of Fig. 1 . The $t_{2 g}$ combination is crucial to describe the minority $\mathrm{Fe}-\mathrm{O}-\mathrm{Mo}$ delocalized electron, which is related to both the halfmetallic and ferrimagnetic character of $\mathrm{Sr}_{2} \mathrm{FeMoO}_{6}$.

In a first ionic approximation, the transition metals ions in $\mathrm{Sr}_{2} \mathrm{FeMoO}_{6}$ are in a $3 \mathrm{~d}^{5} 4 \mathrm{~d}^{1}$ state. ${ }^{22}$ The ground state is expanded in terms of $3 \mathrm{~d}^{5+n} \underline{L}^{n+m} 4 \mathrm{~d}^{1+m}$ covalently mixed configurations, where $\underline{\mathrm{L}}$ denotes a symmetry-adapted $\mathrm{O} 2 \mathrm{p}$ ligand hole. All the possible charge transfer configurations are included explicitly in the ground state expansion. Non-local charge fluctuations such as $3 \mathrm{~d}^{5} 4 \mathrm{~d}^{1} \rightarrow 3 \mathrm{~d}^{4} 4 \mathrm{~d}^{2}$ and $3 \mathrm{~d}^{5} 4 \mathrm{~d}^{1} \rightarrow 3 \mathrm{~d}^{6} 4 \mathrm{~d}^{0}$ are also contemplated. They can be achieved by an indirect secondorder process via the $\mathrm{O} 2 \mathrm{p}$ orbitals, for instance: $3 \mathrm{~d}^{5} 4 \mathrm{~d}^{1} \rightarrow$ $3 \mathrm{~d}^{6} \underline{\mathrm{L}} 4 \mathrm{~d}^{1} \rightarrow 3 \mathrm{~d}^{6} 4 \mathrm{~d}^{0}$.

There are two complete sets of model parameters for each transition metal octahedron: the Coulomb repulsion $U$, the $\mathrm{p}-\mathrm{d}$ charge transfer energy $\Delta$, the core-hole potential $Q(Q=U / 0.83)$, and the pd $\sigma$ charge transfer integral $T_{\sigma}\left(T_{\pi}=-T_{\sigma} / 2\right) \cdot{ }^{17}$ These parameters are given with respect to the average of the multiplet of each configuration. The multiplet splittings of each $3 \mathrm{~d}^{5+n} \underline{\mathrm{L}}^{n+m} 4 \mathrm{~d}^{1+m}$ charge transfer configuration are given in terms of the crystal field parameter $10 D q$, the set of Kanamori parameters $u, u^{\prime}$, and $j$, as well as the p-p hybridization $p p \sigma-$ $p p \pi \cdot{ }^{17}$ The main model parameters and the multiplet parameters for the $\mathrm{FeO}_{6}$ and $\mathrm{MoO}_{6}$ octahedra are given in Table 1. These parameters follow the expected chemical trend, ${ }^{17,18}$ and are consistent with those in related compounds. ${ }^{22-24}$

The calculation of the different spectral weights is performed in three steps. First, the Hamiltonian matrix $\hat{H}$ is diagonalized to obtain the ground state $\left|\psi_{0}\right\rangle$. Then, the corresponding Green function $G(\omega)$ is calculated using the following expression:

$$
G(\omega)=\left\langle\psi_{0}\left|\hat{O}^{\dagger} \frac{1}{\omega-\hat{H}+i \Gamma / 2} \hat{O}\right| \psi_{0}\right\rangle,
$$

where $\hat{O}$ is the appropriate operator for each experimental technique. For the valence band photoemission spectrum, $\hat{O}$ annihilates the $\mathrm{Fe} 3 \mathrm{~d}$, Mo $4 \mathrm{~d}$ and $\mathrm{O} 2 \mathrm{p}$ valence electrons. For the core level photoemission spectra, $\hat{O}$ annihilates Fe $2 \mathrm{p}$ and Mo

Table 1 Model parameters used in the double cluster calculation. The main parameters are: the Coulomb repulsion $U$, the $p-d$ charge transfer energy $\Delta$, and the pd $\sigma$ charge transfer integral $T_{\sigma}$. The multiplet parameters are: the crystal field parameter $10 D q$, the exchange interaction $j$, and the $p p \sigma-p p \pi$ hybridization. All values are given in $\mathrm{eV}$

\begin{tabular}{ccc}
\hline Parameter & $\mathrm{Fe}^{3+}$ & $\mathrm{Mo}^{5+}$ \\
\hline$U$ & 7.5 & 2.6 \\
$\Delta$ & 3.0 & 6.1 \\
$T_{\sigma}$ & 2.1 & 3.9 \\
$10 D q$ & 1.3 & 3.3 \\
$j$ & 0.8 & 0.6 \\
$p p \sigma-p p \pi$ & 1.0 & 1.0
\end{tabular}


$3 p$ core electrons. Finally, the corresponding spectral weight function $A(\omega)$ is obtained using the standard formula:

$$
A(\omega)=-\frac{1}{\pi} \operatorname{Im} G(\omega),
$$

The entire calculation procedure is implemented using the built in facilities of the Quanty package..$^{25-27}$ The single cluster model calculations of the $\mathrm{FeO}_{6}$ and $\mathrm{MoO}_{6}$ octahedra were performed in the same way and using the same set of parameters.

\section{Results and discussion}

\subsection{Ground state properties}

The occupancy of the different configuration in the ground state of $\mathrm{Sr}_{2} \mathrm{FeMoO}_{6}$ are listed in Table 2. The dominant contribution is given by the charge transfer configurations $3 \mathrm{~d}^{5} \underline{\mathrm{L}} 4 \mathrm{~d}^{2}$ and $3 \mathrm{~d}^{6} \underline{\mathrm{L}} 4 \mathrm{~d}^{1}$, which are followed by the base ionic configuration $3 \mathrm{~d}^{5} 4 \mathrm{~d}^{1}$. The relatively large occupancy of the charge transfer configurations indicates a great degree of covalent bonding. The calculated occupation of the Fe $3 \mathrm{~d}$ orbitals in the ground state is 5.5 electrons, whereas the calculated occupation of the Mo $4 \mathrm{~d}$ levels is 1.6 electrons. These occupations are larger than the expected $\mathrm{Fe}^{3+}\left(3 \mathrm{~d}^{5}\right)$ and $\mathrm{Mo}^{5+}\left(4 \mathrm{~d}^{1}\right)$ ionic values, which signals the importance of the Fe $3 \mathrm{~d}-\mathrm{O} 2 \mathrm{p}$ and Mo $4 \mathrm{~d}-\mathrm{O} 2 \mathrm{p}$ hybridization. The relevance of hybridization is also revealed by the relatively large occupancy of the double charge transfer configuration $3 \mathrm{~d}^{6} \underline{\mathrm{L}}^{2} 4 \mathrm{~d}^{2}$.

The relatively large percentage of the $3 d^{6} 4 d^{0}$ configuration indicates the importance of non-local Mo-O-Fe charge fluctuations. This configuration is not achieved by the direct $3 d^{5} 4 d^{1}$ $\rightarrow 3 \mathrm{~d}^{6} 4 \mathrm{~d}^{0}$ process, but rather by the indirect $3 \mathrm{~d}^{5} 4 \mathrm{~d}^{1} \rightarrow 3 \mathrm{~d}^{6} \underline{\mathrm{L}} 4 \mathrm{~d}^{1}$ $\rightarrow 3 \mathrm{~d}^{6} 4 \mathrm{~d}^{0}$ process. On the other hand, the opposite non-local Fe-O-Mo charge transfer $3 \mathrm{~d}^{4} \mathrm{~d}^{2}$ configuration shows a relatively small occupation. This charge fluctuation is strongly suppressed due to the relatively large exchange stabilization of the Fe $3 d^{5}$ electrons. It is worth noting that the relatively strong $3 \mathrm{~d}^{5} 4 \mathrm{~d}^{1} \rightarrow 3 \mathrm{~d}^{6} 4 \mathrm{~d}^{0}$ process corresponds to the minority Mo $4 \mathrm{~d}^{1}$ electron, whereas the $3 \mathrm{~d}^{5} 4 \mathrm{~d}^{1} \rightarrow 3 \mathrm{~d}^{4} 4 \mathrm{~d}^{2}$ transition involves the majority $\mathrm{Fe} 3 \mathrm{~d}^{5}$ electrons and is greatly diminished. The

Table 2 Occupancy of the different configurations in the calculated ground state of $\mathrm{Sr}_{2} \mathrm{FeMoO}_{6}$

\begin{tabular}{cc}
\hline Configuration & Occupancy \\
\hline $3 \mathrm{~d}^{5} \underline{\mathrm{L}} 4 \mathrm{~d}^{2}$ & $20.9 \%$ \\
$3 \mathrm{~d}^{6} \underline{L} 4 \mathrm{~d}^{1}$ & $20.0 \%$ \\
$3 \mathrm{~d}^{5} 4 \mathrm{~d}^{1}$ & $19.6 \%$ \\
$3 \mathrm{~d}^{6} \underline{L}^{2} 4 \mathrm{~d}^{2}$ & $15.2 \%$ \\
$3 \mathrm{~d}^{5} \mathrm{~L}^{2} 4 \mathrm{~d}^{3}$ & $7.7 \%$ \\
$3 \mathrm{~d}^{6} 4 \mathrm{~d}^{0}$ & $7.3 \%$ \\
$3 \mathrm{~d}^{6} \underline{L}^{3} 4 \mathrm{~d}^{3}$ & $4.8 \%$ \\
$3 \mathrm{~d}^{5} \underline{\mathrm{L}^{3}} 4 \mathrm{~d}^{4}$ & $1.3 \%$ \\
$3 \mathrm{~d}^{7} \underline{\mathrm{L}}^{2} 4 \mathrm{~d}^{1}$ & $1.2 \%$ \\
$3 \mathrm{~d}^{7} \underline{\mathrm{L}^{3}} 4 \mathrm{~d}^{2}$ & $0.7 \%$ \\
$3 \mathrm{~d}^{6} \underline{\mathrm{L}^{4}} 4 \mathrm{~d}^{4}$ & $0.7 \%$ \\
$3 \mathrm{~d}^{7} \underline{\mathrm{L}} 4 \mathrm{~d}^{0}$ & $0.5 \%$ \\
$3 \mathrm{~d}^{4} 4 \mathrm{~d}^{2}$ & $0.1 \%$
\end{tabular}

opposite behavior of the minority vs. majority charge fluctuations is in agreement with the half-metallic character of $\mathrm{Sr}_{2} \mathrm{FeMoO}_{6}$.

\subsection{Fe 2p core level spectroscopy}

Fig. 2 shows the experimental Fe 2p core level photoemission spectrum of $\mathrm{Sr}_{2} \mathrm{FeMoO}_{6}$ (dots). The Fe 2p peak is split by spinorbit interaction into the $\mathrm{Fe} 2 \mathrm{p}_{3 / 2}$ and $\mathrm{Fe} 2 \mathrm{p}_{1 / 2}$ contributions about 711 and $724 \mathrm{eV}$, respectively. In turn, these peaks are followed by broad charge transfer satellites at around $718 \mathrm{eV}$ and $731 \mathrm{eV}$.

Fig. 2 also compares the experimental result to the calculated core level spectral weight (solid line). The top panel displays the results of the double cluster calculation, whereas the bottom panel compares to the single cluster model results. The discrete contributions were broadened with Gaussian and Lorentzian broadenings to account for the experimental resolution and the

\section{Fe 2p Core Level Spectroscopy}

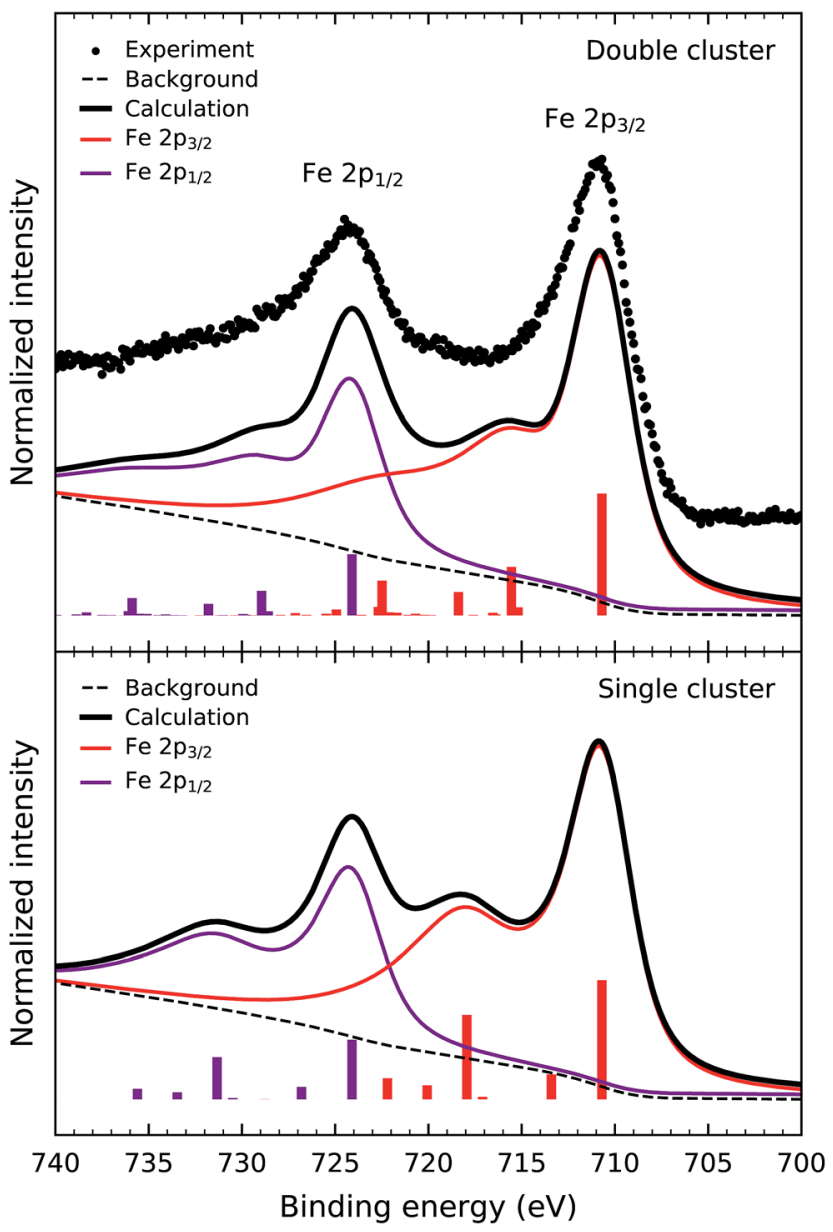

Fig. 2 Fe $2 p$ core level photoemission spectrum of $\mathrm{Sr}_{2} \mathrm{FeMoO}_{6}$ (dots) compared to the calculated spectral weight (solid line) of the double and single cluster models. The experimental spectrum presents the Fe $2 p_{3 / 2}$ and $\mathrm{Fe} 2 \mathrm{p}_{1 / 2}$ components and the respective charge transfer satellites. The calculated spectrum is dominated by well screened $\mathrm{Fe}$ $2 p^{5} 3 d^{6} L$ states and exhibits satellite structures of Fe $2 p^{5} 3 d^{7} L^{2}$ character. 
core hole lifetime. Finally, an integral background was added to the calculated curve to take into account inelastic processes.

The calculated spectral weight of the double cluster model reproduces satisfactorily the experimental data. The main peaks are due to the well screened Fe $2 p^{5} 3 d^{6} \underline{L}$ states, whereas the satellite structures are composed of Fe $2 \mathrm{p}^{5} 3 \mathrm{~d}^{7} \underline{L}^{2}$ states. These charge transfer satellites in the core level spectrum represent a many-body effect, which reflects the highly correlated nature of the $\mathrm{Fe}^{3+}\left(3 \mathrm{~d}^{5}\right)$ ion in $\mathrm{Sr}_{2} \mathrm{FeMoO}_{6}\left(U>T_{\sigma}\right)$. The energy position and relative intensity of the satellites are sensitive to the $U, \Delta$ and $T_{\sigma}$ parameters. On the other hand, the single cluster calculation overestimates the intensity of the charge transfer satellites, mainly because it does not include the non-local screening contribution which comes from the $\mathrm{MoO}_{6}$ octahedra.

\subsection{Mo 3p core level spectroscopy}

Fig. 3 shows the experimental Mo 3p core level photoemission spectrum of $\mathrm{Sr}_{2} \mathrm{FeMoO}_{6}$ (dots). The Mo 3p peaks are split by

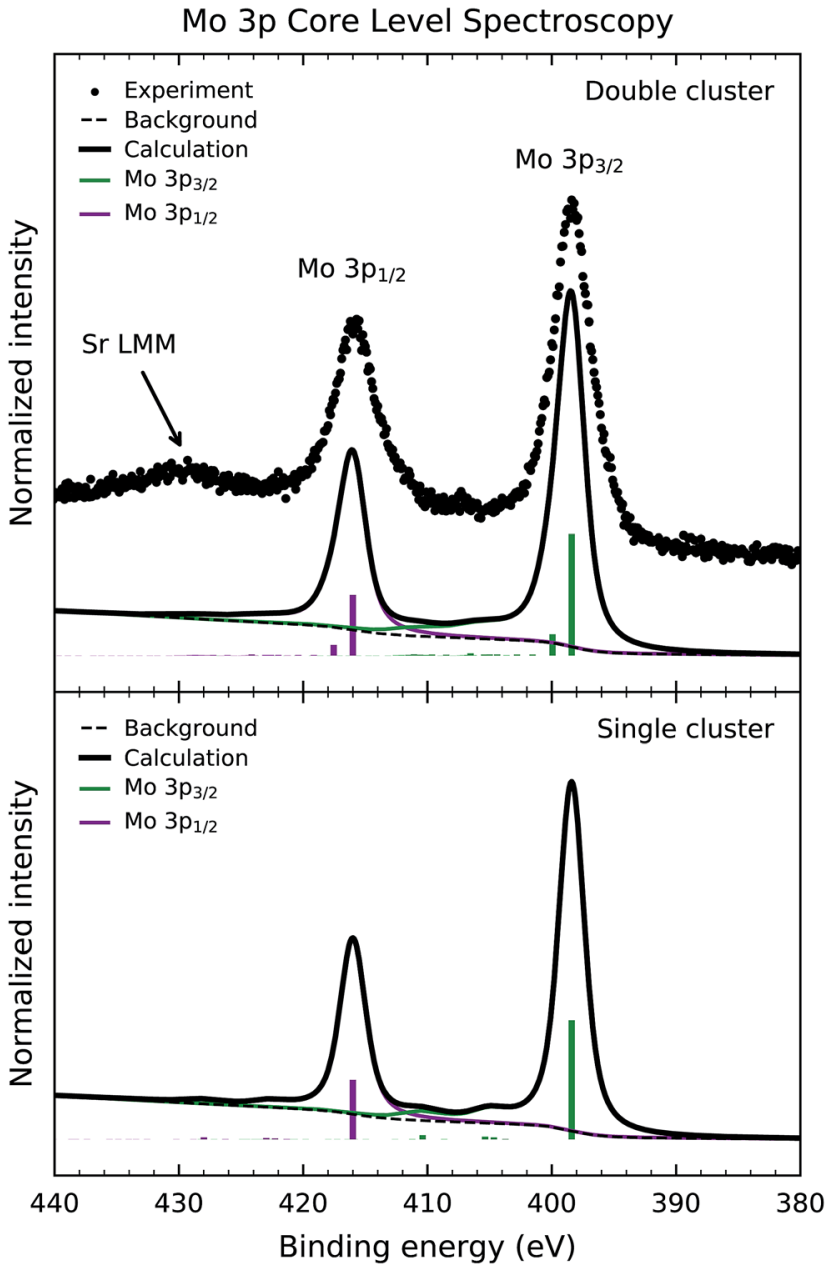

Fig. 3 Mo 3p core-level photoemission spectrum of $\mathrm{Sr}_{2} \mathrm{FeMoO}_{6}$ (dots) compared to the calculated spectral weight (solid line) of the double and single cluster models. The experimental spectrum presents the Mo $3 p_{3 / 2}$ and $M o 3 p_{1 / 2}$ peaks, while the structure around $430 \mathrm{eV}$ is due to $\mathrm{Sr}$ LMM Auger decay. The calculated spectrum is dominated by states with Mo $3 p^{5} 4 d^{1}$ character. spin-orbit interaction into the Mo $3 \mathrm{p}_{3 / 2}$ and Mo $3 \mathrm{p}_{1 / 2}$ contributions around 398 and $416 \mathrm{eV}$, respectively. In this case, the core level spectrum does not exhibit a prominent satellite structure. Finally, the experimental data presents an accidental superposition with the Sr LMM Auger electron decay around $430 \mathrm{eV}$.

Fig. 3 also compares the experimental data to the calculated core-level spectral weight (solid line) of the double and single cluster models. Likewise, the discrete contributions were also broadened to account for the experimental resolution and the core hole lifetime. In the same way, the integral background was calculated and added to the final calculated curve.

The calculated spectral weight of the double cluster calculation successfully reproduces the peaks in the experimental result. The leading structure at $398 \mathrm{eV}$ arises from mixed Mo $3 \mathrm{p}^{5} 4 \mathrm{~d}^{2} \underline{\mathrm{L}}$ and $3 \mathrm{p}^{5} 4 \mathrm{~d}^{1}$ final states, while the smaller contribution at $400 \mathrm{eV}$ has mainly Mo $3 \mathrm{p}^{5} 4 \mathrm{~d}^{1}$ character. The smaller satellite structure, in this case, is attributed to the less correlated character of the $\mathrm{Mo}^{5+}\left(4 \mathrm{~d}^{1}\right)$ ion in $\mathrm{Sr}_{2} \mathrm{FeMoO}_{6}\left(U<T_{\sigma}\right)$. For this reason, the calculated results are less sensitive to the values of the model parameters. In this case, the single cluster calculation reproduces reasonably well the experimental spectrum, mainly because the charge transfer processes are less important than in the Fe $2 p$ core level spectrum.

\subsection{Valence band spectroscopy}

Fig. 4 presents the experimental valence band photoemission spectrum of $\mathrm{Sr}_{2} \mathrm{FeMoO}_{6}$ (dots). The dashed line denotes the Fermi level energy $E_{\mathrm{F}}$. The spectrum is composed of three main regions: the Fe 3d-O 2p-Mo $4 \mathrm{~d}$ mixed states close to the Fermi level, the non-bonding and bonding $\mathrm{O} 2 \mathrm{p}$ band from 9 to $4 \mathrm{eV}$, and the Fe $3 \mathrm{~d}$ charge transfer satellites from 15 to $9 \mathrm{eV}$.

Fig. 4 also compares the experimental data to the calculated spectral weight (solid line) using double and single cluster model calculations. The individual contributions of the Fe 3d, Mo $4 \mathrm{~d}$ and $\mathrm{O} 2 \mathrm{p}$ states are presented below. The intensity of the peaks were weighted with the appropriate photoelectron crosssections for a photon energy of $1840 \mathrm{eV}$. The discrete peaks were convoluted with a Gaussian function to account for the experimental resolution. Finally, an integral background was added to take into account inelastic scattering effects. The single cluster result was calculated using a linear combination of the $\mathrm{FeO}_{6}$ and $\mathrm{MoO}_{6}$ single clusters.

The calculated spectral weight of the double cluster model is in good agreement with the experimental valence band photoemission data. The first feature around $1.0 \mathrm{eV}$ is attributed to Fe 3d-O 2p-Mo 4d mixed states; these are related to the non-local charge fluctuations which give rise to the conducting behavior of $\mathrm{Sr}_{2} \mathrm{FeMoO}_{6}$. The Fe $3 \mathrm{~d}$ states are split by crystal field effects into the $\mathrm{Fe} t_{2 \mathrm{~g}}$ and $\mathrm{Fe} e_{\mathrm{g}}$ contributions, and appear about 5.5 and $4.0 \mathrm{eV}$, respectively. The non-bonding $\mathrm{O} 2 \mathrm{p}$ band corresponds to O 2 p states which are not hybridized with Fe $3 d$ and Mo $4 d$ states, and shows up between 4.0 and $8.0 \mathrm{eV}$ (purple line). On the other hand, the bonding $\mathrm{O} 2 \mathrm{p}$ band corresponds to $\mathrm{O} 2 \mathrm{p}$ states which are hybridized with Fe $3 \mathrm{~d}$ and Mo $4 \mathrm{~d}$ states, and arises from 6.0 and $10.0 \mathrm{eV}$ (blue line). Finally, the charge 


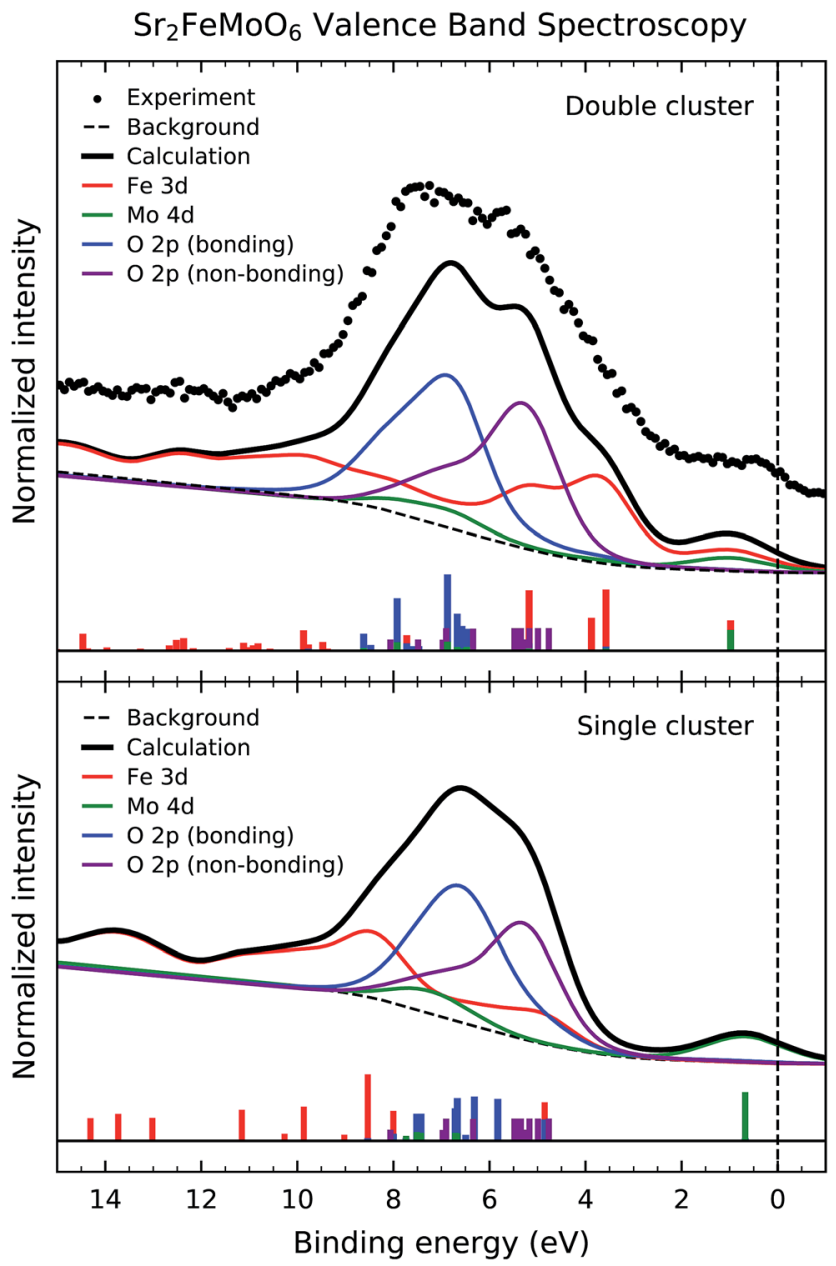

Fig. 4 Valence band photoemission spectrum of $\mathrm{Sr}_{2} \mathrm{FeMoO}_{6}$ (dots) compared to the calculated spectral weight (solid line) of the double and single cluster models. The experimental spectrum is composed of Fe $3 d-O 2 p-M o ~ 4 d$ mixed states close to the Fermi level, nonbonding and bonding $\mathrm{O} 2 \mathrm{p}$ band from 9 to $4 \mathrm{eV}$, and the Fe $3 \mathrm{~d}$ charge transfer satellites from 15 to $9 \mathrm{eV}$. The first feature around $1 \mathrm{eV}$ is attributed to Fe $3 d-O 2 p-M o 4 d$ mixed states, while the bottom of the valence band is dominated by charge transfer satellites related to the Fe 3d states.

transfer satellites, mostly related to the Fe $3 \mathrm{~d}$ states, emerge above $9.0 \mathrm{eV}$. We note that the Fe 3d-O 2p and Mo 4d-O 2p hybridization are crucial to describe the features in the valence band spectrum. Further, the correlation effects of the Fe 3d states is essential to explain the charge transfer satellites at high binding energies.

The Mo 4d spectral weight (green line) presents a peak around $1.0 \mathrm{eV}$ as well as structure about $8.0 \mathrm{eV}$. The former is related to the $\mathrm{Fe} 3 \mathrm{~d}-\mathrm{O} 2 \mathrm{p}-\mathrm{Mo} 4 \mathrm{~d}$ mixed states, whereas the later is attributed to Mo $4 \mathrm{~d}-\mathrm{O} 2 \mathrm{p}$ hybridization. The energy position and relative intensity of these Mo $4 \mathrm{~d}$ structures are in agreement with those observed in a recent Mo $\mathrm{L}_{3}$ resonant photoemission experiment. ${ }^{21}$

Although the single cluster model results reproduce the overall shape of the valence band, they fail to explain the magnetic order and the electrical conductivity of the compound. The double cluster model gives an anti-parallel ordering of the Fe $3 \mathrm{~d}^{5}$ and Mo $4 \mathrm{~d}^{1}$ magnetic moments, which is in agreement with the observed ferrimagnetic ordering in $\mathrm{Sr}_{2} \mathrm{FeMoO}_{6}$, whereas the single cluster model produces an independent alignment of the magnetic moments yielding a paramagnetic state. Further, the double cluster model indicates the importance of the non-local Fe 3d-O 2p-Mo 4d fluctuations, which are related to the half-metallic character of this oxide, whereas the single cluster model only consider the local Fe 3d-O 2p and Mo 4d-O 2p fluctuations and gives rise to an insulating state.

The non-local charge fluctuations are reflected not only in the composition of the ground state, but also contribute to the individual transitions in the experimental spectra. In fact, the first removal state in the valence band spectra, around $1 \mathrm{eV}$, is a non-local Fe $3 \mathrm{~d}-\mathrm{O} 2 \mathrm{p}-\mathrm{Mo} 4 \mathrm{~d}$ mixed state, according to the double cluster, but it is a local Mo $4 \mathrm{~d}-\mathrm{O} 2 \mathrm{p}$ state in the single cluster calculation. Therefore, the double cluster calculation is crucial to explain not only the physical properties of $\mathrm{Sr}_{2} \mathrm{FeMoO}_{6}$, but also the character of the spectral features present in the valence band spectrum.

\section{Summary and conclusions}

In summary, we studied the electronic structure of $\mathrm{Sr}_{2} \mathrm{FeMoO}_{6}$ using bulk sensitive photoemission spectroscopy. The experimental data were analyzed using configuration interaction cluster model calculations. The charge transfer $3 \mathrm{~d}^{5} \underline{\mathrm{L}} 4 \mathrm{~d}^{2}$ and $3 \mathrm{~d}^{6} \underline{\mathrm{L}} 4 \mathrm{~d}^{1}$ configurations dominate the ground state; this indicates a large degree of $\mathrm{Fe}-\mathrm{O}$ and $\mathrm{Mo}-\mathrm{O}$ hybridization in this compound. The occupancy of the $3 \mathrm{~d}^{6} 4 \mathrm{~d}^{0}$ configuration shows the importance of non-local Fe-O-Mo charge fluctuations; this configuration is achieved through the indirect $3 \mathrm{~d}^{5} 4 \mathrm{~d}^{1} \rightarrow$ $3 \mathrm{~d}^{6} \underline{\mathrm{L}} 4 \mathrm{~d}^{1} \rightarrow 3 \mathrm{~d}^{6} 4 \mathrm{~d}^{0}$ process. This non-local charge fluctuation occurs in the minority spin channel, whereas the transitions in the majority spin sector are strongly suppressed; which is in accordance with the half-metallic character attributed to the $\mathrm{Sr}_{2} \mathrm{FeMoO}_{6}$ compound. The Fe $2 \mathrm{p}$ core level photoemission spectrum shows rather large charge transfer satellites. These satellites are related to many-body effects caused by the highly correlated nature of the Fe $3 d$ levels. On the other hand, these effects are smaller in the Mo $3 p$ core level spectrum due to the less correlated character of the Mo $4 \mathrm{~d}$ states. Charge transfer satellites are also observed in the valence band spectrum and are again associated to the Fe $3 \mathrm{~d}$ electrons. Although the single cluster model calculations are able to reproduce the experimental spectra, they fail to explain the physical properties of the $\mathrm{Sr}_{2} \mathrm{FeMoO}_{6}$ compound. In this context, the double cluster model calculations are crucial to explain both the ferrimagnetic ordering and the half-metallic character of this compound. To conclude, the metal-oxygen hybridization, non-local Fe-O-Mo charge fluctuations, and many-body effects are all relevant topics in the electronic structure of $\mathrm{Sr}_{2} \mathrm{FeMoO}_{6}$.

\section{Conflicts of interest}

There are no conflicts to declare. 


\section{Acknowledgements}

The authors would like to thank the Coordenação de Aperfeiçoamento de Pessoal de Nível Superior (CAPES) and Fundação Araucária do Paraná for their financial support.

\section{References}

1 K.-I. Kobayashi, T. Kimura, H. Sawada, K. Terakura and Y. Tokura, Nature, 1998, 395, 677-680.

2 Y. Tomioka, T. Okuda, Y. Okimoto, R. Kumai, K.-I. Kobayashi and Y. Tokura, Phys. Rev. B, 2000, 61, 422-427.

3 L. Balcells, J. Navarro, M. Bibes, A. Roig, B. Martínez and J. Fontcuberta, Appl. Phys. Lett., 2001, 78, 781-783.

4 A. Gupta and J. Z. Sun, J. Magn. Magn. Mater., 1999, 200, 2443.

5 M. Ziese, Rep. Prog. Phys., 2002, 65, 143.

6 D. Serrate, J. M. De Teresa, P. A. Algarabel, C. Marquina, J. Blasco, M. R. Ibarra and J. Galibert, J. Phys.: Condens. Matter, 2007, 19, 436226.

7 D. D. Sarma, P. Mahadevan, T. Saha-Dasgupta, S. Ray and A. Kumar, Phys. Rev. Lett., 2000, 85, 2549-2552.

8 M. S. Moreno, J. E. Gayone, M. Abbate, A. Caneiro, D. Niebieskikwiat, R. D. Sánchez, A. de Siervo, R. Landers and G. Zampieri, Solid State Commun., 2001, 120, 161-164.

9 K. Kuepper, I. Balasz, H. Hesse, A. Winiarski, K. C. Prince, M. Matteucci, D. Wett, R. Szargan, E. Burzo and M. Neumann, Phys. Status Solidi A, 2004, 201, 3252-3256.

10 T. Saitoh, M. Nakatake, A. Kakizaki, H. Nakajima, O. Morimoto, S. Xu, Y. Moritomo, N. Hamada and Y. Aiura, Phys. Rev. B: Condens. Matter Mater. Phys., 2002, 66, 035112.

11 J.-S. Kang, J. H. Kim, A. Sekiyama, S. Kasai, S. Suga, S. W. Han, K. H. Kim, T. Muro, Y. Saitoh, C. Hwang, C. G. Olson, B. J. Park, B. W. Lee, J. H. Shim, J. H. Park and B. I. Min, Phys. Rev. B: Condens. Matter Mater. Phys., 2002, 66, 113105.

12 J. Navarro, J. Fontcuberta, M. Izquierdo, J. Avila and M. C. Asensio, Phys. Rev. B: Condens. Matter Mater. Phys., 2004, 69, 115101.

13 S. Ray, A. Kumar, D. D. Sarma, R. Cimino, S. Turchini, S. Zennaro and N. Zema, Phys. Rev. Lett., 2001, 87, 097204.
14 K. Kuepper, M. Kadiroglu, A. V. Postnikov, K. C. Prince, M. Matteucci, V. R. Galakhov, H. Hesse, G. Borstel and M. Neumann, J. Phys.: Condens. Matter, 2005, 17, 4309-4317. 15 M. Besse, V. Cros, A. Barthélémy, H. Jaffrès, J. Vogel, F. Petroff, A. Mirone, A. Tagliaferri, P. Bencok, P. Decorse, P. Berthet, Z. Szotek, W. M. Temmerman, S. S. Dhesi, N. B. Brookes, A. Rogalev and A. Fert, Europhys. Lett., 2002, 60, 608-614.

16 G. van der Laan, C. Westra, C. Haas and G. A. Sawatzky, Phys. Rev. B, 1981, 23, 4369-4380.

17 A. E. Bocquet, A. Fujimori, T. Mizokawa, T. Saitoh, H. Namatame, S. Suga, N. Kimizuka, Y. Takeda and M. Takano, Phys. Rev. B: Condens. Matter Mater. Phys., 1992, 45, 1561-1570.

18 M. Imada, A. Fujimori and Y. Tokura, Rev. Mod. Phys., 1998, 70, 1039-1263.

19 E. B. Guedes, M. Abbate, K. Ishigami, A. Fujimori, K. Yoshimatsu, H. Kumigashira, M. Oshima, F. C. Vicentin, P. T. Fonseca and R. J. O. Mossanek, Phys. Rev. B: Condens. Matter Mater. Phys., 2012, 86, 235127.

20 M. Abbate, F. C. Vicentin, V. Compagnon-Cailhol, M. C. Rocha and H. Tolentino, J. Synchrotron Radiat., 1999, 6, 964-972.

21 H. P. Martins, F. Prado, A. Caneiro, F. C. Vicentin, R. J. O. Mossanek and M. Abbate, Europhys. Lett., 2017, 118, 37002 .

22 H. P. Martins, F. Prado, A. Caneiro, F. C. Vicentin, D. S. Chaves, R. J. O. Mossanek and M. Abbate, J. Alloys Compd., 2015, 640, 511-516.

23 M. Abbate, G. Zampieri, J. Okamoto, A. Fujimori, S. Kawasaki and M. Takano, Phys. Rev. B: Condens. Matter Mater. Phys., 2002, 65, 165120.

24 V. Stoeberl, M. Abbate, L. M. S. Alves, C. A. M. dos Santos and R. J. O. Mossanek, J. Alloys Compd., 2017, 691, 138-143.

25 M. W. Haverkort, M. Zwierzycki and O. K. Andersen, Phys. Rev. B: Condens. Matter Mater. Phys., 2012, 85, 165113.

26 M. W. Haverkort, G. Sangiovanni, P. Hansmann, A. Toschi, Y. Lu and S. Macke, Europhys. Lett., 2014, 108, 57004.

27 Y. Lu, M. Höppner, O. Gunnarsson and M. W. Haverkort, Phys. Rev. B: Condens. Matter Mater. Phys., 2014, 90, 085102. 